\title{
NUTRITION AND CLIMATIC STRESS
}

$\mathrm{N}_{\text {tivied }}$

UTRITION and environmental physiology are both applied studies, each operating on a particular aspect of and parts of the organism, and each using for its premises the purer sciences of physiology and biochemistry. Having the same bases the two subjects have, therefore, much ground in common. Too frequently that ground is cultivated by the two groups, lacking not so much common interest as common knowledge. Some appreciable step towards breaking down specialist compartmentalism at this particular boundary was made by the symposium on "Nutrition and Climatic Stress" held by the Nutrition Society on April 26 at the Hannah Dairy Research Institute in Ayr.

While research on nutrition is a steady flood and represents a considerable fraction of biological research, work on human environmental physiology has had a hesitant history, often dependent on the impetus lent by wars and rumours of wars, and the grants in aid that go with them. On the other hand, work both on the nutrition and on the responses to environmental stress in farm animals has been steadily progressing in close co-ordination, without benefit of such alarms and excursions. The location of this meeting was particularly well chosen, for work at the Hannah Institute on the nutrition and environmental physiology of cattle has been proceeding for many years. The facilities, techniques and community of interest now assembled there for this work form an impressive example of integrated research. The demonstrations of climatic chambers and respiration calorimeters for cattle formed one of the most important services of the meeting.

Dr. T. Bedford (London School of Hygiene and Tropical Medicine) in his introductory address indicated a problem which recurred in various forms throughout the meeting - the problem of measurement and of scales of measurement. Nearly all the thermal components of climate (air temperature, humidity, wind velocity) can be measured with fair precision; even radiant heat can be measured under some limited conditions. From these measurements, however, no satisfactory index of climate as a physiological environment, even with respect only to its effect on heat dissipation, has so far been devised. This difficulty of scale of measurement recurs in the measurement of physiological response where different functions respond at different temperatures, and any one function responds, in different species, at different temperatures and even in different ways (for example, the heat loss by evaporation in sweating and nonsweating mammals). It recurs yet again in assessment of nutrition, where a scale that takes account of the interaction of environmental temperature and energy exchange may differ markedly from one assessing exchange of protein or other food factors.

H. E. Lewis and J. P. Masterton (Medical Research Council Laboratories) spoke on some aspects of the feeding of field expeditions in cold climates. The primary problem here is that of creating a palatable ration of low weight and bulk and high energy value. A most interesting secondary aspect of this, however, is the problem of food packaging in convenient units and in such a way as to be at the same time resistant to the casual impacts of shipping and handling, and yet yielding without struggle when the time comes actually to get at the food.

The conditions of living of the explorers of the British North Greenland Expedition, 1952-54, as shown in a film and as apparent from some of the published records of the expedition, well illustrate a further point raised by Dr. Bedford, that any consideration of the stress of climate must take into account the indoor as well as the outdoor climate. The effective climate to which the members of this expedition were exposed during the winter months was that of a house maintained at a rather higher temperature $\left(50-70^{\circ} \mathrm{F}\right.$.) than that obtaining in the majority of British homes during the same season. It came as little surprise, therefore, to find that the explorers put on weight during this period.

The most obvious interaction of climatic stress and nutrition is in the field of energy exchange. It is, indeed, only this aspect of the subject on which any considerable body of data is available. Perhaps because of this it is also the field in which some major disagreements and discrepancies are apparent. R. E. Johnson and F. Sargent (University of Illinois) described in outline the results of a marathon study on U.S. Air Force personnel in hot-humid, temperate and cold environments, covering 8,696 man-days. This work involved, as variables, climate, food intake, food composition, water intake, and work. Since some of their date on imposed stress and physiological response were entirely qualitative and much was largely qualitative, the interpretation of this large mass of information is a difficult and equivocal business. Apart from the inter-relations which might be expected from well-known physiological principles, they found that water-balance under any given set of conditions was a maximum at a particular osmotic load, and declined on either side of it. They also found that low temperatures or a high fat diet without a high imposed work load can produce a degree of ketosis which is directly related to the percentage of fat in the diet and inversely related to the temperature.

Johnson and Sargent interpreted their energy data to mean that at a low temperature there is little difference from thermal neutrality in the partition of energy usage from protein, fat and carbohydrate. They also tried to explain some of their findings on nitrogen-balance and intake of energy on the basis of the specific dynamic action of the various rations and the energy available for muscular work at various temperatures.

K. L. Blaxter (Hannah Institute) presented data to show that, at low temperatures, the cow derives almost all its excess heat production from metabolism of fat. He also demonstrated that below the critical temperature, with respect to energy exchange (namely, thermal neutrality), the heat increment of feeding becomes inseparable from general heat production. In addition, however, he pointed out that the critical temperature varies with the level of feeding, falling, in some animals, even to $0^{\circ} \mathrm{C}$. or below. The critical environmental temperature also, of course, varies with heat insulation, and in man that includes clothing. While, therefore, many of these discrepancies may lie in the lack of 
comparability of experimental conditions and subjects, the fact that such discrepancies can exist in the only reasonably well-documented part of the subject illustrates how little has yet been done.

The great differences in response to climatic stress between a sweating and a nearly non-sweating mammal were made very plain by J. D. Findlay (Hannah). In a dry atmosphere, increase in environmental temperature well above normal deep-body temperature in man can be tolerated by great increases in rate of sweating and elimination of heat by evaporation (the classical experiment proved survival at $127^{\circ}$ C.). The cow has a type of poorly vascularized sweat gland, but approaches its sweating maximum at about $26^{\circ} \mathrm{C}$., above which loss of heat by panting is substituted. Panting in turn reaches its maximum effectiveness at about $40^{\circ} \mathrm{C}$., which is an almost lethal temperature.

Information on other aspects of interaction of climatic stress and nutrition is sparse. Discussion by R. H. Fox (Medical Research Council Laboratories) and by Blaxter and others on the changes in requirement for vitamins, and on losses of amino-acids and minerals through the skin, and the relation of these to such deficiency-diseases as pellagra and kwashiorkor were speculative.
S. D. Morrison

\section{X-RAY RESEARCH}

$\mathrm{T}$ HE Spring Conference of the X-ray Analysis Group of the Institute of Physics was held in the College of Science and Technology, Manchester, on April 18 and 19. No particular subject was fixed for discussion, and papers were invited on any topic in the field of crystallography and X-ray analysis.

A novel feature of the Conference was a partsession devoted to the reading of five papers by research students. Although there have, of course, been papers at previous conferences of which research students have been authors or joint authors, the Group committee felt that to set aside a period for such papers might particularly encourage research students to feel that their work was of value and worthy of the attention of the Group as a whole. The five papers discussed were selected from some ten papers offered.

Mr. J. V. P. Long (Cavendish Laboratory, Cambridge) described experiments on the use of fine-focus $X$-ray tubes and X-ray absorption and emission spectra for quantitative micro-analysis. Although some information can occasionally be obtained from absorption measurements by using one $\mathrm{X}$-ray wavelength alone, such measurements are more fruitful if two wave-lengths are employed lying on each side of an absorption edge of the element of interest. An apparatus developed for such measurements makes use of an X-ray focal spot about $1 \mu$ in diameter and a crystal monochromator with a proportional counter as detector of the transmitted radiation. It has been used for the determination of calcium in rock sections and in biological materials. Emission spectroscopy, with a somewhat similar apparatus except that the monochromator is now placed out of the primary X-ray beam, has also been used for determination of calcium in these specimens; it seems likely to be more sensitive to small amounts of a given element than the absorption technique, but to be less well fitted to provide accurate information about the distribution of the element in a thin section.

The remaining four papers were concerned with structural investigations. Mr. R. Maitland (King's College, Newcastle upon Tyne) dealt with the crystal structures of chromous and chromic fluorides. The trifluoride structure was determined with little difficulty from powder photographs; it is rhombohedral and of the vanadium trifluoride type, with regular $\mathrm{CrF}_{6}$ octahedra. In chromium difluoride, however, the symmetry is only monoclinic, and the $\mathrm{CrF}_{6}$ octahedra are distorted, giving three different chromium-fluorine bond-lengths ; the structure may be regarded as a distortion of a rutile-type structure.
The differences in bonding between the two fluorides can be completely accounted for by ligand field theory.

Mr. S. Hosoya (University College, Cardiff) discussed the structures of two isomers of thianthrene dioxide, which are of interest as examples of sulphoxides, about which there has been much recent controversy. Only two isomers of thianthrene dioxide have been found ; the $\alpha$-isomer, which has the higher melting point and smaller dipole moment of the two, has been shown by two-dimensional X-ray studies to have the anti-cis form as predicted by Taylor' ${ }^{1}$ and the $\beta$-isomer to have the trans form. Three-dimensional studies of both isomers are in progress to provide detailed information about the sulphur bonding and the folding of the molecules for comparison with other sulphoxides.

Mr. R. Hine (University College, Cardiff) described a determination of the crystal structure of $(+) \mathrm{S}$. methyl-L-cysteine-S-oxide. This substance crystallizes as colourless orthorhombic laths; the space group is $P 2_{1} 2_{2} 2_{1}$, with $a=5 \cdot 2, b=7 \cdot 4, c=16 \cdot 3 \mathrm{~A}$. and the molecular arrangement consists of pairs of sheets parallel to (001) with an elaborate arrangement of short non-bonded distances between certain atoms of the molecules in each sheet. The structure contains an optically active carbon of known configuration, and thus the determination is, in effect, one of the absolute configuration of an asymmetric sulphur atom. The structure of the (-) S-isomer is now being investigated to provide further information about the $\mathrm{S}-\mathrm{O}$ bond.

Mr. H. C. Watson (College of Science and Technology, Manchester) had made use of the optical transform techniques developed in the Physics Department of the College in determining the structure of $\beta$-naphthol from X-ray data. The space group of the crystal is $I a$, and the unit cell has $a=8 \cdot 185, b=5.950, c=36.29$ A., with $\beta=119^{\circ}$ 52 . Projections parallel to the 100 and to the 010 axis were calculated independently; these two projections left a choice between only two passible structures, the projections parallel to 100 and 010 of which were identical but different parallel to 001 . The choice between them was made on the basis of intermolecular distances, an oxygen-oxygen distance of $1.8 \mathrm{~A}$. in one structure being rejected in favour of $2.7 \mathrm{~A}$. in the other, and verified by examining the intensities of a number of general reflexions $h k l$ in an oscillation photograph about the $b$-axis.

R. L. GORDON

1 Taylor, T. W. J., J. Chem. Soc., 625 (1935). 